\title{
Principles of developing of a scintillation detector based on SiPM for field gamma-spectrometry problems
}

Ilya Bokaty, Valery Korotaev, Victor Denisov, Victoria Ryzhova, Vladimir Aseev

Ilya Bokaty, Valery Korotaev, Victor Denisov, Victoria Ryzhova, Vladimir Aseev, "Principles of developing of a scintillation detector based on SiPM for field gamma-spectrometry problems," Proc. SPIE 11367, Photosensitive Materials and their Applications, 113671I (1 April 2020); doi: $10.1117 / 12.2556078$

SPIE. Event: SPIE Photonics Europe, 2020, Online Only 


\title{
Principles of developing of a scintillation detector based on SiPM for a field gamma-spectrometry problems
}

\author{
Ilya Bokaty*a, Valery Korotaev ${ }^{\mathrm{a}}$, Victor Denisov ${ }^{\mathrm{b}}$, Victoria Ryzhova ${ }^{\mathrm{a}}$, Vladimir Aseev ${ }^{\mathrm{a}}$ \\ ${ }^{a}$ ITMO University, Saint-Petersburg, Russia, ${ }^{\text {b }}$ Flagman-geo Ltd, Saint-Petersburg, 197376, Russian \\ Federation
}

\begin{abstract}
Spectral methods for the detection and analysis of ionizing radiation sources are widely used in many fields of science and technology. Ecology, geology and mineralogy, metallurgy and the chemical industry, the oil industry and medicine this is not the whole list of areas where the gamma-ray spectrometer acts as an independent software and hardware tool. Scintillation spectrometers are used to monitor the radiation environment, to search for sources of ionizing radiation, tomography and much more. However, this method is based on the use of vacuum photoelectronic multipliers (PMTs), which complicates the use of devices in the field.
\end{abstract}

This article presents an analysis of gamma-ray scintillation spectrometers built on the basis of silicon photoelectronic multipliers (SiPM). The fields of application of field gamma spectrometers are considered and the basic requirements that the devices must meet to ensure the successful solution of the tasks are determined. The basic principles of the development of field scintillation gamma spectrometers that take into account the processes and transformations of the optical range radiation inside the detector are described. Moreover, the article describes personal experience in the development of an experimental sample of a field gamma spectrometer based on SiPM.

In this article, the use of field device refers to any use outside of laboratory conditions.

Keywords: scintillation, gamma-spectrometry, SiPM

\section{INTRODUCTION}

Gamma spectrometers are one of the most common methods of non-destructive testing of radioactive materials, due to the possibility of isotopic analysis of samples containing a complex mixture of radionuclides, and the possibility of a quantitative description of their activity and mass. From the point of view of the principle of recording ionizing radiation, gamma spectrometers can be divided into solid-state, magnetic, gas-filled and scintillation detectors.

A typical example of solid-state detectors are devices based on semiconductor materials - most often germanium or silicon [1]. Using instruments of this class it is possible to obtain energy spectra with high resolution (of the order of fractions of a percent of the full width at half maximum (FWHM) [2]). However, the operation of such detectors should be accompanied by cooling in cryostats to the temperature of liquid nitrogen. Therefore, solid-state spectrometers are rarely used in the field. It is also worth noting that the price of a highly purified germanium (HPGe) detector will be an order of magnitude higher than the price of a field scintillation detector.

Detectors in which ionizing radiation interacts with a scintillation crystal are a fundamentally different class. Under the influence of ionizing radiation on the atoms of the scintillation crystal, the atoms go into an excited state. Returning to the ground state, the scintillator atoms emit photons in the optical range, which are detected by the photodetector. A feature of the scintillation method is the high detection efficiency of gamma rays (up to 100\%) and low energy resolution $(>10 \%)$ [3]. The advantage of such detectors is the ability to create mobile devices for field use.

For a long time, photomultiplier tubes (PMT) were used as a photodetector in such systems. PMTs have high sensitivity and low inertia, which allows us to create accurate equipment on their basis [4] for recording and analyzing fast processes. However, PMTs have a few disadvantages that make it difficult to use devices based on them, especially in the field. PMTs consists of a photocathode, a system of dynodes and an anode in a glass flask; therefore, the device has

*bokatyi.ilia@bk.ru; phone+79215577899

low resistance to mechanical interactions and high weight and size characteristics. Another negative factor is the need for

Photosensitive Materials and their Applications, edited by Robert R. McLeod, Inmaculada Pascual Villalobos,

Yasuo Tomita, John T. Sheridan, Proc. of SPIE Vol. 11367, 113671I · ( 2020 SPIE

CCC code: $0277-786 \mathrm{X} / 20 / \$ 21 \cdot$ doi: $10.1117 / 12.2556078$ 
a high voltage power supply to create high potential at accelerating dynodes. The high voltage source is large and introduces significant interference into the measurements of the WEPF [5].

Modern application requirements require the use of brand-new detectors that provide minimum weight and size characteristics, a high level of automation, multichannel, wireless connectivity and the ability to integrate into large systems [6].

The development of the production of solid-state photoelectronic receivers has made silicon photomultipliers (SiPM) a commercially available version of the sensing element in scintillation spectrometry devices. It is worth noting that, in addition to a fundamentally new mechanism for detecting the flow of optical radiation, SiPM are free from the disadvantages of PMTs.

Despite significant advantages, there are practically no serial SiPM-based gamma-ray scintillation spectrometers on the world market, but there are a large number of experimental devices. Table 1 provides an overview of the most illustrative examples of the implementation of scintillation gamma spectrometers based on SiPM.

Table 1. Examples of developed scintillation detectors.

\begin{tabular}{|c|c|c|c|c|c|c|c|c|}
\hline \multirow[t]{2}{*}{ Ref. } & \multirow[t]{2}{*}{ Application } & \multicolumn{2}{|c|}{ Scintillator } & \multicolumn{2}{|c|}{ Receiver } & \multirow{2}{*}{$\begin{array}{c}\begin{array}{l}\text { Light } \\
\text { guide }\end{array} \\
\text { Shape, } \\
\text { Length }\end{array}$} & \multicolumn{2}{|c|}{ Parameters } \\
\hline & & Material & Size & Type & Active area & & $\begin{array}{l}\text { FWHM } \\
\text { for }{ }^{137} \mathrm{Cs}\end{array}$ & $\begin{array}{l}\text { Linearity } \\
\text { as R2 }\end{array}$ \\
\hline [7] & $\begin{array}{l}\text { Personal } \\
\text { monitoring }\end{array}$ & Ce:GAGG & $3 \times 3 \times 20 \mathrm{~mm}^{3}$ & Hamamatsu & $3 \times 3 \mathrm{~mm}^{2}$ & No & 5.8 & 0.9924 \\
\hline [8] & $\begin{array}{l}\text { Experimental } \\
\text { sample }\end{array}$ & $\mathrm{NaI}(\mathrm{Tl})$ & $\varnothing 25 \times 25 \mathrm{~mm}^{3}$ & $\begin{array}{l}\text { SensL } \\
\text { MicroFC }\end{array}$ & $12 \times 12 \mathrm{~mm}^{2}$ & $\begin{array}{l}\text { Conoid, } \\
10 \mathrm{~mm}\end{array}$ & 7.7 & NA \\
\hline [8] & $\begin{array}{l}\text { Experimental } \\
\text { sample }\end{array}$ & $\mathrm{NaI}(\mathrm{Tl})$ & $\varnothing 50 \times 50 \mathrm{~mm}^{3}$ & $\begin{array}{l}\text { SensL } \\
\text { MicroFC, }\end{array}$ & $12 \times 12 \mathrm{~mm}^{2}$ & $\begin{array}{l}\text { Conoid, } \\
10 \mathrm{~mm}\end{array}$ & 10.2 & NA \\
\hline [9] & $\begin{array}{l}\text { Sample for } \\
\text { nuclear } \\
\text { physics } \\
\text { experiments }\end{array}$ & $\mathrm{LaBr} 3: \mathrm{Ce}$ & $\varnothing 25 \times 25 \mathrm{~mm}^{3}$ & $\begin{array}{l}\text { FBK } \\
\text { (NUV-HD) }\end{array}$ & $31.9 \times 38.9 \mathrm{~mm}^{2}$ & No & 3.7 & NA \\
\hline [10] & $\begin{array}{l}\text { Experimental } \\
\text { sample }\end{array}$ & $\mathrm{CsI}(\mathrm{Tl})$ & $6 \times 6 \times 10 \mathrm{~mm}^{3}$ & $\begin{array}{l}\text { SensL } \\
\text { ArrayJ }\end{array}$ & $6 \times 6 \mathrm{~mm}^{2}$ & No & 7.9 & $\begin{array}{l}\text { On the } \\
\text { chart in } \\
\text { the } \\
\text { source }\end{array}$ \\
\hline [11] & $\begin{array}{l}\text { Sample for } \\
\text { radiation } \\
\text { monitoring }\end{array}$ & $\mathrm{NaI}(\mathrm{Tl})$ & $\varnothing 50 \times 50 \mathrm{~mm}^{3}$ & $\begin{array}{l}\text { SensL } \\
\text { ArrayJ }\end{array}$ & $12 \times 12 \mathrm{~mm}^{2}$ & No & 9.8 & $\begin{array}{l}\text { On the } \\
\text { chart in } \\
\text { the } \\
\text { source }\end{array}$ \\
\hline$[11]$ & $\begin{array}{l}\text { Sample for } \\
\text { radiation } \\
\text { monitoring }\end{array}$ & $\mathrm{NaI}(\mathrm{Tl})$ & $\varnothing 50 \times 50 \mathrm{~mm}^{3}$ & $\begin{array}{l}\text { SensL } \\
\text { ArrayJ }\end{array}$ & $12 \times 12 \mathrm{~mm}^{2}$ & $\begin{array}{l}\text { Conoid, } \\
10 \mathrm{~mm}\end{array}$ & 11.3 & $\begin{array}{l}\text { On the } \\
\text { chart in } \\
\text { the } \\
\text { source }\end{array}$ \\
\hline$[11]$ & $\begin{array}{l}\text { Sample for } \\
\text { radiation } \\
\text { monitoring }\end{array}$ & $\mathrm{NaI}(\mathrm{Tl})$ & $\varnothing 50 \times 50 \mathrm{~mm}^{3}$ & $\begin{array}{l}\text { SensL } \\
\text { ArrayJ }\end{array}$ & $12 \times 12 \mathrm{~mm}^{2}$ & $\begin{array}{l}\text { Conoid, } \\
30 \mathrm{~mm}\end{array}$ & 11.8 & $\begin{array}{l}\text { On the } \\
\text { chart in } \\
\text { the } \\
\text { source }\end{array}$ \\
\hline [12] & $\begin{array}{l}\text { Experimental } \\
\text { sample }\end{array}$ & CLYC & $1000 \mathrm{~mm}^{3}$ & Hamamatsu & $6 \times 6 \mathrm{~m}^{2}$ & No & 8.3 & 0.994 \\
\hline $\begin{array}{l}\text { This } \\
\text { work }\end{array}$ & $\begin{array}{l}\text { Experimental } \\
\text { sample for } \\
\text { geo } \\
\text { exploration }\end{array}$ & CsI(Tl) & $\begin{array}{l}14 \times 14 \times 28 \\
\mathrm{~mm}^{3}\end{array}$ & $\begin{array}{l}\text { SensL } \\
\text { ArrayC }\end{array}$ & $14 \times 14 \mathrm{~mm}^{2}$ & No & 7.8 & 0.996 \\
\hline
\end{tabular}


It is worth noting the general interest in creating an inexpensive, compact, wireless system that performs real-time measurements [10] [13]. In all the works presented in Table 1 (except the first and last), the output face of the scintillation crystal is larger than the SiPM's active area. The main contributing factor to FWHM is the scintillation crystal material. In the case indicated in the source [11], it is worth noting insignificant deterioration of FWHM when lightguides are included in the system.

\section{RESULTS}

The vast majority of articles presented on the subject of scintillation gamma spectrometry were written by specialists in the field of nuclear engineering without due interest in the optical processes occurring inside the recording path of the gamma spectrometer.

The probability of detecting a gamma quantum by a scintillation crystal depends on the path length of the gamma quantum in the scintillation medium. To increase the probability of a gamma ray incident on the photodetector, it is preferable to form photons near its sensitive area. Therefore, the shape of the crystal must simultaneously provide the longest path length of the gamma ray and the smallest path length of optical photons.

From the data given in Table 1, it follows that in most gamma spectrometers, when detecting uncollimated gamma-ray beams, the sides of the crystal are selected in the ratio 1:1:1 (Figure 1 (a)) or 1:1:2 (Figure 1 (b)).

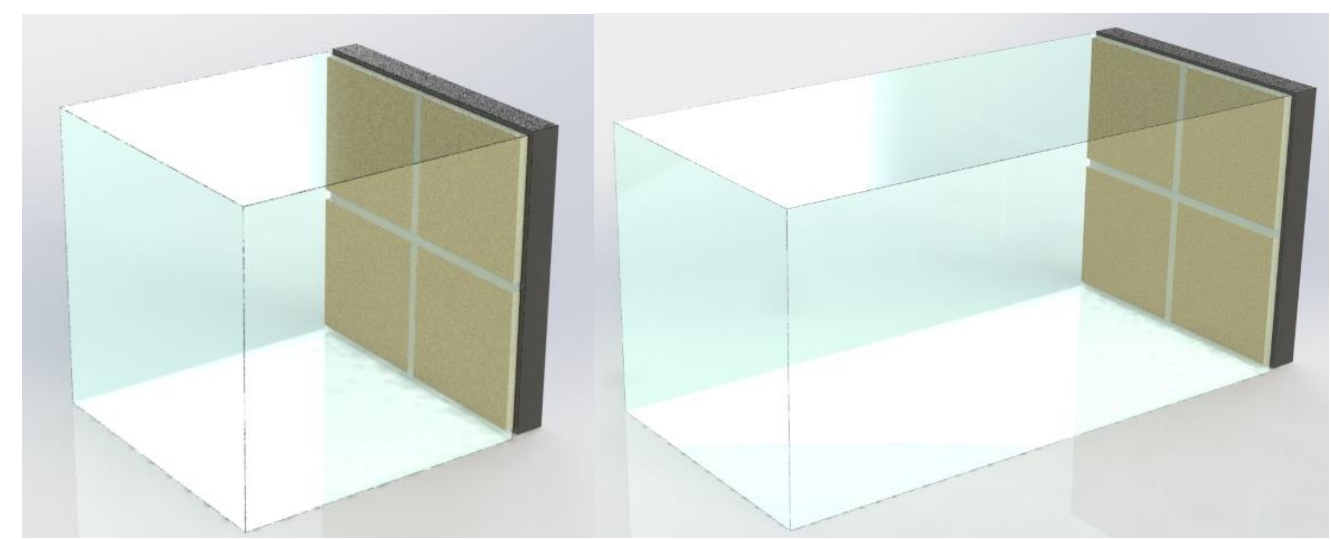

(a)

(b)

Figure 1. Model of crystals of different proportions with SiPM docked to the output face. The ratio of crystal faces 1:1:1 (a) and $1: 1: 2(b)$.

Large-volume scintillators are used to increase the efficiency of gamma-ray registration. Therefore, for efficient registration of photons in the optical range, it is necessary to use SiPM with a large sensitive surface equal to the output face of the scintillator. Such SiPMs are generally high cost or consist of a smaller set of SiPMs, which also increases the cost of the device.

In such cases, to increase the efficiency of detecting photons in the optical range, conical optical fibers are used (Figure 2) with an input face that repeats the shape of a scintillation crystal and output in the form of a SiPM sensitive area. The main factors affecting the optical loss of photons are the length of the fiber, the angle of inclination of the generatrix of the cone, the material of the fiber and the reflective coating of its faces.

An important condition for a reasonable choice of detector elements is the matching of the spectral characteristics of the radiation of the scintillation crystal and the spectral characteristics of the sensitivity of the photodetector [14]. In addition, it is necessary to coordinate the power range of the optical radiation of the crystal and the dynamic range of linearity of the photodetector.

The efficiency of scintillator radiation using a photodetector (SiPM) can be described by the following formula: 


$$
k=\frac{\int_{\lambda_{1}}^{\lambda_{2}} \Phi_{\lambda}(\lambda) \cdot S(\lambda) d \lambda}{\int_{0}^{\infty} \Phi_{\lambda}(\lambda) d \lambda}
$$

where $\Phi_{\lambda}(\lambda)$ - is the spectral distribution function of the scintillator radiation flux, $S(\lambda)$ - is the spectral sensitivity of SiPM.

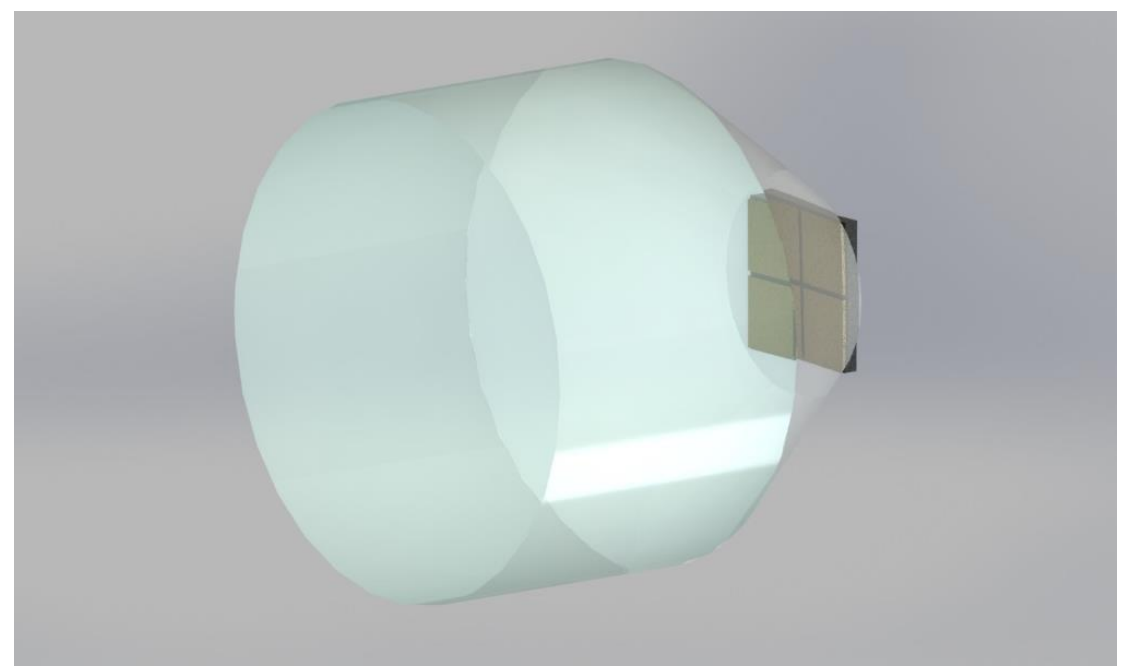

Figure 2. Model of a cylindrical crystal docked with SiPM through a conical lightguide.

In the course of this work, the spectral sensitivity characteristic of SiPM SensL ArrayC-60035 was experimentally investigated. The structural diagram of the stand for studying the spectral characteristics of SiPM is shown in Figure 3.

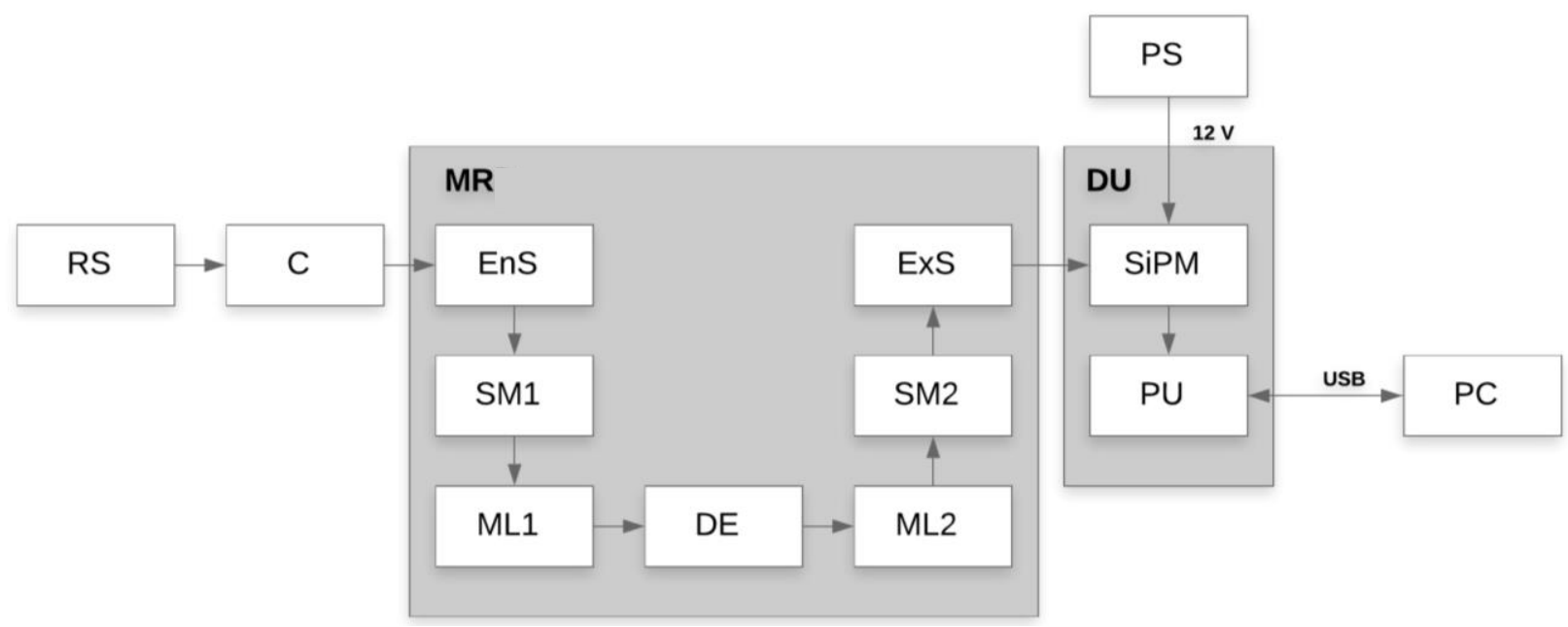

Figure 3. Scheme of the stand for the research of spectral characteristics. RS - radiation source, C - condenser,

MR - monochromator, EnS - entrance slit, SM1,SM2 - swivel mirrors, DE - dispersing element, ML1,ML2 - mirror lenses,

ExS - exit slit, DU - detector unit, SiPM - silicon photoelectronic multiplier, PU - processing unit, PS - power supply,

$\mathrm{PC}$ - personal computer.

The measurements were carried out using a monochromatic radiation shaper operating according to the Czerny-Turner principle with a planar emitting diode as a radiation source and a diffraction grating as a dispersing element. The study of 
spectral characteristics was carried out in the range of 300-1000 nm with a step of $35 \mathrm{~nm}$. The plot of the reduced spectral sensitivity of the SMPM is shown in Figure 4 (a).

A graph of the spectral distribution of scintillator radiation is shown in Figure 1 (b). Thus, the efficiency of using a pair of CsI(Tl) scintillator and SiPM ArrayC-60035 was $k=0.729$. This coefficient makes it possible to numerically estimate the recorded part of the scintillator radiation.

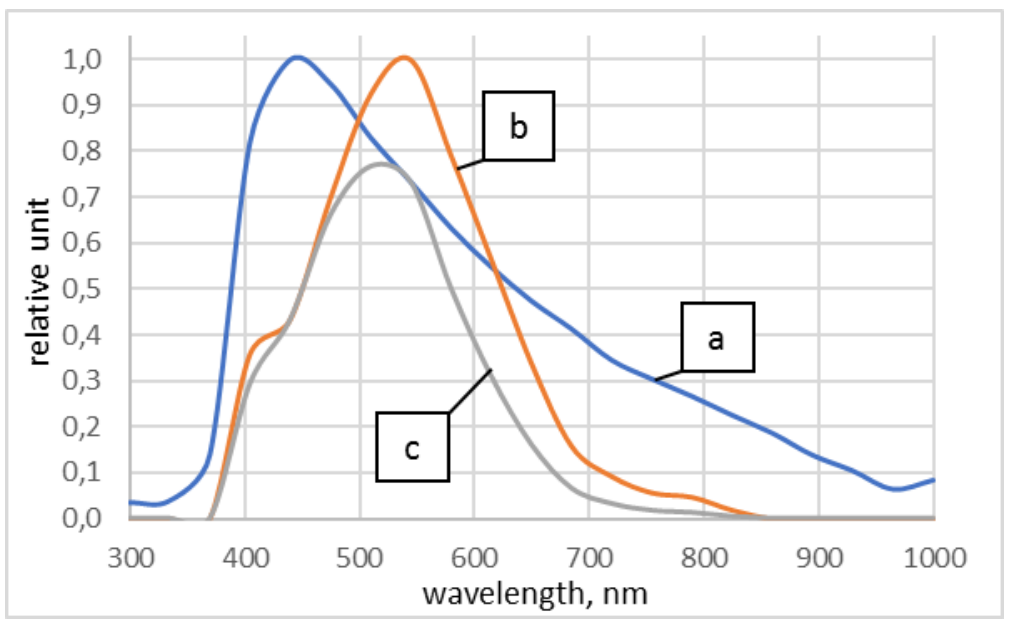

Figure 4. Graphs for calculating the coefficient of efficiency of using a pair of scintillator CsI(Tl) and SiPM ArrayC-60035. (a) plot of the reduced spectral sensitivity of SiPM ArrayC-60035; (b) plot of the relative spectral distribution of the CsI(Tl) scintillator; (c) plot of the spectral efficiency of registration of photons in the optical range $k(\lambda)$.

The above parameters allow us not to exceed the maximum value of the radiation flux of the optical range incident on SiPM, which should be selected from the operating conditions of the receiving device on a linear portion of its energy characteristic [15]. The conversion function of the measuring device remains linear if the signal at the SiPM output is proportional to the radiation intensity from scintillation at the sensitive area of the receiver. In an ideal situation, each photon created in a scintillation crystal triggers only one microcell of a silicon photomultiplier; therefore, the energy of the particle absorbed by the scintillation crystal will be proportional to the number of triggered SiPM microcells.

The number of triggered SiPM cells is described by the formula [16]:

$$
N_{\text {fired }}=M\left(1-\exp \left(-\frac{P D E \cdot N_{p h}}{M}\right)\right)
$$

where $N_{\text {fired }}$ - is the number of triggered microcells; $M$ - is the total number of microcells of SIPM; PDE - the integrated photon detection efficiency of the optical range, depends on the wavelength of the optical radiation, the geometric filling factor of SiPM (the ratio of the sum of the areas of insensitive areas of SiPM to the total area of SiPM); $N_{p h}-$ is the number of photons incident on the active region of the matrix. Thus, it is possible to vary the number of photons incident on the SiPM active region by influencing the dynamic range of detected photons, and as a result, the accuracy of determining the energy of gamma rays.

The number of photons formed in the scintillator material during the absorption of gamma rays depends on the energy of the absorbed gamma ray and the characteristics of the scintillation crystal used. However, not all photons arising from scintillation reach the sensitive SiPM site, since some of the photons are absorbed inside the crystal and when reflected from crystal faces. In addition, photons are reflected at interfaces in the conjugation region of the scintillation crystal and $\mathrm{SiPM}$. Reflection depends on the angle of incidence of radiation at the interface. These factors determine the effectiveness of the structure containing the crystal scintillator and SiPM.

As part of this work, a research of the dynamic range of SiPM was made. During the study, the signal measured by SiPM was measured at various values of the radiation flux generated by the planar emitting diode. The measurements were 
carried out in the range of the radiation flux from $4.9 \cdot 10^{-3} \mathrm{~mW}$ to $12.8 \mathrm{~mW}$. Within the specified range, 10 series of measurements were performed. The measured value was the volt signal taken from the SiPM output and characterizing the number of triggered receiver cells during photon registration.

Figure 5 shows the normalized energy characteristic of $\operatorname{SiPM} w\left(\Phi_{e}\right)$, where $w$-is the volt signal value at the value of the flux $\Phi_{e}$. From the obtained dependence it follows that the linearity range of the energy characteristic of the SiPM under study corresponds to the range of the radiation source power from 0.4 to $2.0 \mathrm{~mW}$.

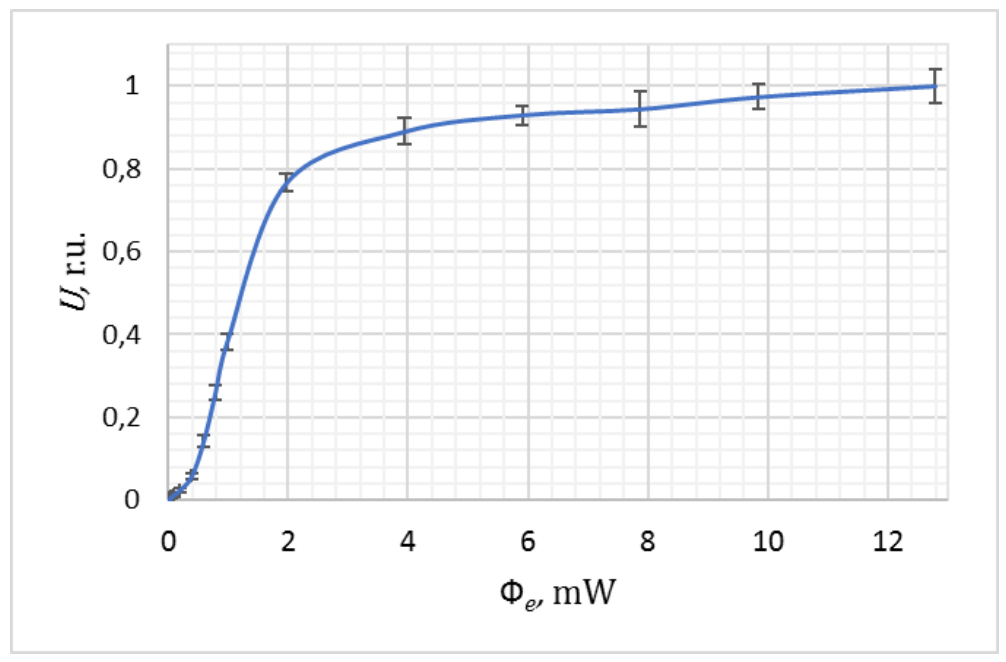

Figure 5. Energy characteristic of SiPM.

From Figure 5, it follows that the SiPM output signal enters saturation at a certain value of the photon flux density. However, even with a radiation flux in the linear range of the energy characteristic, there is a possibility that two photons will fall into the same SiPM microcell with a time difference shorter than the recovery time of this cell. As a result of this, one of the photons will not be registered.

Studies have been conducted of the distribution of photons over the SiPM site as a result of scintillation caused by a single gamma quantum. Under simulation conditions, the scintillation energy was set in relative units. A CsI(Tl) crystal with 5 reflective faces was considered. The sixth face was in direct contact with the SiPM ArrayC-60035 entrance window. The flow distribution was modeled in Zemax. The simulation result of the flow distribution over one of the sensitive sites is shown in Figure 6.

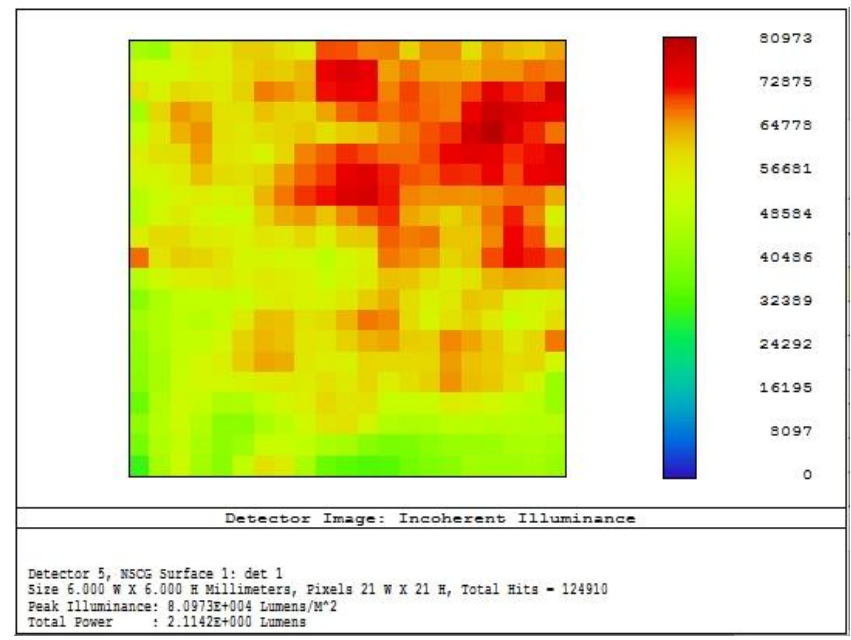

Figure 6. Energy distribution at the detector site. The peak value is red and equals 81000 relative units. 


\section{CONCLUSION}

In this paper, we considered the basic principles for constructing a SiPM scintillation detector for field gamma spectrometry. It has been shown that it is advisable to create field gamma spectrometers based on scintillation detectors and SiPM as photodetectors. The preferred geometric ratios of scintillation crystals used in known implementations are determined. Lightguide implementation options are considered that make it possible to increase the optical efficiency of the structure. The coordination of the spectral characteristics of the scintillator radiation and the spectral sensitivity of the $\mathrm{SiPM}$ is considered. Experimentally investigated the spectral characteristics of the sensitivity of the SiPM. The efficiency of a combination of a scintillation detector and SiPM was determined. The linearity range of the signal conversion in the photodetector is determined.

\section{ACKNOWLEDGMENTS}

This work was financially supported by Government of the Russian Federation, Grant (08-08).

\section{REFERENCES}

[1] Wallbrink, P. J., D. E. Walling, and Q. He. "Radionuclide measurement using HPGe gamma spectrometry." Handbook for the assessment of soil erosion and sedimentation using environmental radionuclides. Springer, Dordrecht, 67-96 (2002).

[2] MEASUREMENT TYPE DESCRIPTION "Gamma-spectrometer based on a detector made of highly pure germanium with electromechanical cooling RKG-1", Appendix to certificate No. 55340 on type approval of measuring instruments (2014).

[3] K. Siegbahn, ed. [Alpha-, Beta, and Gamma-Ray Spectroscopy], North-Holland Publ., Amsterdam, p. 1639 (1965).

[4] Anisimova I. I., Glukhovskoy B. M., [Photoelectronic Multipliers], Sov. Radio, Moscow, (1974).

[5] Berkovsky A. G., Gavanin V. A., Zaydel I. N., [Vacuum photoelectronic devices], Energy, Moscow, (1976).

[6] Denisov V.M., Korotaev V.V., Titov A.B., Blokhina A.A., Kleshchenok M.A., "Overview of field gamma spectrometries based on Si-photomultiplier" Proceedings of SPIE, № Vol. 10231, (2017).

[7] Park, Hye Min, and Koan Sik Joo. "Development and performance characteristics of personal gamma spectrometer for radiation monitoring applications." Sensors 16.6: 919, (2016) .

[8] Huang, Tuchen, et al. "NaI (Tl) scintillator read out with SiPM array for gamma spectrometer." Nuclear Instruments and Methods in Physics Research Section A: Accelerators, Spectrometers, Detectors and Associated Equipment 851: 118-124, (2017).

[9] Cozzi, Giulia, et al. "High-Resolution Gamma-Ray Spectroscopy With a SiPM-Based Detection Module for 1" and 2" LaBr 3: Ce Readout." IEEE Transactions on Nuclear Science 65.1, 645-655 (2017).

[10] Becker, Eric M., et al. "Small prototype gamma spectrometer using CsI (Tl) scintillator coupled to a solid-state photomultiplier." IEEE Transactions on Nuclear Science 60.2, 968-972 (2013).

[11] Kim, Junhyeok, et al. "Efficient design of a $\emptyset 2 \times 2$ inch NaI (Tl) scintillation detector coupled with a SiPM in an aquatic environment." Nuclear Engineering and Technology 51.4, 1091-1097 (2019).

[12] Mesick, Katherine E., et al. "Performance of several solid state photomultipliers with CLYC scintillator." 2015 IEEE Nuclear Science Symposium and Medical Imaging Conference (NSS/MIC). IEEE (2015).

[13] David, S., and I. Kandarakis. "Development of a Submillimeter Portable Gamma-Ray Imaging Detector, Based on a GAGG: $\mathrm{Ce}$-Silicon Photomultiplier Array." International Conference on Engineering of Scintillation Materials and Radiation Technologies. Springer, Cham (2018).

[14] Otte, Adam Nepomuk, et al. "Characterization of three high efficiency and blue sensitive silicon photomultipliers." Nuclear Instruments and Methods in Physics Research Section A: Accelerators, Spectrometers, Detectors and Associated Equipment 846, 106-125 (2017).

[15] Dvornikov O. V., Chekhovsky V. A., Dyatlov V. L. [Means of registration of pulsed visible radiation of low intensity. Part 1. Features and capabilities of multichannel photodetectors with internal amplification. Overview], Instruments and measurement methods (2012).

[16] Poleshchuk R.V. [Development of photon methods for the experimental complex of the Center for Underground Physics CUPP.] Moscow (2015). 